\title{
Application Research of Residue Theorem in Integral Calculation
}

\author{
Haijian Cui ${ }^{1, a}$, Wenyao Xiong ${ }^{1, b}$ \\ ${ }^{1}$ Nanchang Institute of Science \& Technology, Nanchang, 330108, China

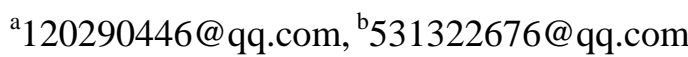

Keywords: residue theorem; integral calculation; infinite integral

\begin{abstract}
This paper, first introduces the method of calculating the residue, and describes and proves the main contents of the residue theorem, and the promotion of the residue theorem. This paper discusses the calculating problems of integral in analysis with the theorem of residue, obtains the general commutation method and formula by using analytical skills, and then makes it easier to resolve the calculating problems.
\end{abstract}

\section{Introduction}

As everyone knows, in the mathematical analysis and practical application, often need to calculate the definite integral or improper integral [1]. The primary function of these points in the integrand, sometimes not expressed by elementary functions, or even can find the original function, if used in mathematical analysis to calculate the integral method are very limited and cumbersome. It is necessary to find a new method. For example, you can consider the real integration into complex integration, to take advantage of the complex integral theory, and the residue theorem is an important tool in this field. We will focus on the complex integral calculation method using the residue theorem function. The basic idea is to integral a section of the realistic function in the real axis, we are in the appropriate additional a curve to form a simple closed curve, which will be transformed into the integral of complex function the contour integral, and then use the residue theorem can be solved. The residue is a complex one of the important basic concepts in function theory, and the analytical function in the isolated singularity Laurent expansion, Cauchy closed composite theorem etc. are closely linked. The residue theorem is complex important theorem in theory of functions, it is complex integral and complex product series to combine, have important applications in practice, especially it can provide a new method for integral calculation, to a certain role in promoting the development of the theory of complex functions. Then the residue theorem can figure out all the points? The answer is negative. The application of residue theorem in integral in also has some limitations. By studying the residue theorem and its application in integration, we can better understand the important theorem of a festival on the integral application. In addition, the application of residue theorem, we can prove the argument principle and important the theorem of Roache theorem, the theorem can be investigated within the area function of the distribution of zeros [2].

\section{Residue and Residue Theorem}

\subsection{Residue}

In the mathematical analysis and practical problems, often require some definite integral or improper integral value of the original function and the integral of the integrand, cannot be expressed by elementary function; sometimes even can find the original function, the calculation is often more complex. In fact, the real integration is always in the interval calculation, and residue theory is a contour integral conclusion. If the calculation of definite integral or improper integral of certain types of using the residue theorem, first try to transform the problem into a contour integral, while only residue calculation of certain analytic functions in the isolated singularities; so, the problem simplifies the calculation. On the other hand, integral or abnormal integral also has some limitations by using the residue theorem, by using the residue to solve integration problems and no universally 
applicable method. Therefore, in this article we consider only a few Integral special types and points out how to calculate simplification for calculating residue problems.

Assume the function $f(z)$ take a finite point as an isolated point, that is $f(z)$ is an analytic function in $0<|z-a|<R, \frac{1}{2 \pi i} \int_{\Gamma} f(z) d z(\Gamma:|z-a|=\rho, 0<\rho<R)$ is the residue of $\mathrm{f}(\mathrm{z})$ in a point, which is recorded as $\operatorname{Res}_{z=a} f(z)[3]$.

\subsection{Residue Theorem}

Through integral calculation using Laurent theorem, the key is to find some ring domain integrand analytic singularities, this can be through the discussion of the integrand is not difficult to determine but need to find the ring domain contains a closed curve, this is not an easy thing, beginners often a headache. Of course, if you find the ring domain that can function to find the Laurent expansion coefficient on the line; and through integral calculation by using residue theorem requires two steps, the first step to find all finite singular point, the second step is to calculate the residue, of course the calculation still need to Laurent singularity about heart function. The use of Laurent's theorem to directly look simple, using the residue theorem to bending, but in fact, because the analytic function area for Laurent expansion is not easy, but not sure the proper area, the need for specific analysis, which makes the direct integral calculation and Laurent theorem is not commonly used; and although the residue theorem is divided into two steps, also need to Laurent started to stay for, but they are in the neighborhood of the singularity to the heart, is to determine the region, but also the development of extension calculation method is more convenient and efficient, because it has clear standards of procedural steps to be followed, the residue theorem in integral calculation is easy to master. In the process of Euler integral, uniform convergence problem of Euler integral can often be tested, may need to introduce the parameter variables to Euler integral derivation in integral number and so on. And the calculation process is very cumbersome to use these methods in this paper. The introduction of residue theorem by the residue theorem to calculate the Euler integral.

Assume the function $f(z)$ is an analytic function in the field of $D$ except $a_{1}, a_{2}, \ldots \ldots, a_{n}$. The function $\mathrm{f}(\mathrm{z})$ is continues in $\mathrm{D}+\mathrm{C}$ except $\mathrm{a}_{1}, \mathrm{a}_{2}, \ldots \ldots, \mathrm{a}_{\mathrm{n}}$, we have:

$$
\int_{c} f(z) d z=2 \pi i \sum_{k=1}^{n} \operatorname{Re}_{z=a_{k}} s f(z)[4]
$$

\subsection{Method to Get Theorem}

We use Residue Theorem to compute the closed integral curve on the value of the problem into the residue calculation each isolated singularity on the problem, in each of the Isolated Singularities of the Laurent expansion in the first negative power coefficient is calculated. In general, for the Laurent expansion is more trouble, because of this, according to the different types of Isolated Singularities, establish some simple methods of residue calculation respectively is very necessary. To apply the residue for integral, should first master the method for the residue. This is a residue in the coefficient of isolated points when we only need to care about the Laurent expansion in the calculation, so the application of Laurent expansion for the residue is the general method. Laurent's theorem is an important content of the series theory, the residue theorem is an important content of the integral theory, integral theorem two can calculate complex function. These beginners often are very confused, which affect the complex function theory to master, following the author answer, so that we focus on the calculation of complex function and integral understanding clear, then through an example to illustrate how these two theorems are integral calculation. Informed by residue theorem, calculation function along the points can be attributed to the calculated contour within the Isolated Singularities of the residue and the residue is also a negative coefficient. A power series of the Laurent singular point, so we only care about the negative power coefficient of the singularity in the Laurent series, that is say, don't calculate the Laurent series can completely determine the residue of the points. 
Theorem. Assume a is the n-order pole of the function $f(z)$, and $f(z)=\frac{\varphi(z)}{(z-a)^{n}}$, in which the function $\varphi(\mathrm{z})$ is analytic in a point and $\varphi(a) \neq 0$, we have:

$$
\operatorname{Res}_{z=a} f(z)=\frac{\varphi^{n-1}(a)}{(n-1) !} .
$$

Inference. Assume a is the two-order pole of the function $\mathrm{f}(\mathrm{z})$, and,$\varphi(z)=(z-a)^{2} f(z)$, we have:

$$
\operatorname{Res}_{z=a} f(z)=\varphi^{\prime}(a) .
$$

\section{Applications of Residue Theorem in Integral Calculation}

Example 1. To calculate $I=\int_{0}^{2 \pi} \frac{d \theta}{1-2 p \cos \theta+p^{2}}(0 \leq|p|<1)$.

Solution: $z=e^{i \theta}$, we have: $d \theta=\frac{d z}{i z}$.

When $p \neq 0$,

$$
1-2 p \cos \theta+p^{2}=1-p\left(z+z^{-1}\right)+p^{2}=\frac{(z-p)(1-p z)}{z},
$$

Therefore, $I=\frac{1}{i} \int_{|z|=1} \frac{d z}{(z-p)(1-p z)}$,

And in the circle of $|z|<1$, we have

$$
f(z)=\frac{1}{(z-p)(1-p z)},
$$

According to the Residue Theorem, we have:

$$
I=\frac{1}{i} \cdot 2 \pi i \cdot \frac{1}{1-p^{2}}=\frac{2 \pi}{1-p^{2}}(0 \leq|p|<1) .
$$

Example 2. Assume a $>0$, to calculate $\int_{0}^{+\infty} \frac{d x}{x^{4}+a^{4}}$.

Solution: As $\int_{0}^{+\infty} \frac{d x}{x^{4}+a^{4}}=\frac{1}{2} \int_{-\infty}^{+\infty} \frac{d x}{x^{4}+a^{4}}$ and $f(z)=\frac{1}{z^{4}+a^{4}}$, has four one-order poles.

$$
a_{k}=a e^{\frac{\pi+2 k \pi}{4} i}(k=0,1,2,3),
$$

which is suitable for the Residue Theorem.

As $\underset{z=a_{k}}{\operatorname{Re} s} f(z)=\left.\frac{1}{4 z^{3}}\right|_{z=a_{k}}=\frac{1}{4 a_{k}^{3}}=\frac{a_{k}}{4 a_{k}^{4}}=-\frac{a_{k}}{4 a^{4}}$,

The function $f(z)$ has only two one-order poles $\mathrm{a}_{0}$ and $\mathrm{a}_{1}$, therefore,

$$
\begin{gathered}
\int_{0}^{+\infty} \frac{d x}{x^{4}+a^{4}}=-\pi i \frac{1}{4 a^{4}}\left(a e^{\frac{\pi}{4} i}+a e^{\frac{3 \pi}{4} i}\right) \\
=-\pi i \frac{1}{4 a^{3}}\left(a e^{\frac{\pi}{4} i}-a e^{-\frac{\pi}{4} i}\right)
\end{gathered}
$$




$$
\begin{gathered}
=\frac{\pi}{2 a^{3}} \sin \frac{\pi}{4} \\
=\frac{\pi}{2 \sqrt{2} a^{3}}
\end{gathered}
$$

Example 3. To calculate $\int_{-\infty}^{+\infty} \frac{x \cos x d x}{x^{2}-2 x+10}$.

Solution: we can easily to check that the function $f(z)=\frac{z e^{i z}}{z^{2}-2 z+10}$ meet the Jordan Theorem, in which $m=1, g(z)=\frac{z}{z^{2}-2 z+10}$.

The one-order poles of the function $\mathrm{f}(\mathrm{z})$ are $z=1+3 i$ and $z=1-3 i$.

$$
\operatorname{Res}_{z=1+3 i} f(z)=\left.\frac{z e^{i z}}{\left(z^{2}-2 z+10\right)^{\prime}}\right|_{z=1+3 i}=\frac{(1+3 i) e^{-3+i}}{6 i} \text {. }
$$

Therefore,

$$
\begin{gathered}
\int_{-\infty}^{+\infty} \frac{x e^{i x} d x}{x^{2}-2 x+10}=2 \pi i \frac{(1+3 i) e^{-3+i}}{6 i} \\
=\frac{\pi}{3} e^{-3}(1+3 i)(\cos 1+i \sin 1) \\
=\frac{\pi}{3} e^{-3}(\cos 1-3 \sin 1)+i \frac{\pi}{3} e^{-3}(3 \cos 1+\sin 1) .
\end{gathered}
$$

We compare the real part and imaginary part of the equation to get:

$$
\begin{aligned}
& \int_{-\infty}^{+\infty} \frac{x \cos x d x}{x^{2}-2 x+10}=\frac{\pi}{3} e^{-3}(\cos 1-3 \sin 1) \\
& \int_{-\infty}^{+\infty} \frac{x \sin x d x}{x^{2}-2 x+10}=\frac{\pi}{3} e^{-3}(3 \cos 1+\sin 1)
\end{aligned}
$$

\section{Conclusion}

The residue theorem and its application played a certain role in promoting the development of the theory of complex functions. It gives some real integral calculation and provides a very effective tool. It is particularly important in this method could not obviously obtain these integral conditions, even though integral method can be used in general under the condition of using residue theorem is generally much easier.

\section{References}

[1] Liang Yikong. A Residue Method of Calculating Infinite Integral [J]. Journal of Shanghai University of Engineering Science, 2015, 29(4): 360-361.

[2] Wang Yulei, Li Caijuan. Calculate a special kind of generalized integral by using residue theorem [J]. Journal of Science of Teachers' College and University, 2016, 36(10): 23-24.

[3] Lu Shengqi. Residue Theory and ts Application [J]. Science \& Technology Information, 2009(33): 947-949.

[4] Li Xiaofei. Calculation of definite integral by residue theorem [J]. Journal of Huanggang Normal University, 2011, 31(6): 34-35. 